\title{
Electromagnetic Scattering by a Finite Strip on a Substrate
}

\author{
Egon Marx \\ National Institute of Standards and Technology, Gaithersburg, MD 20899, USA
}

\section{Introduction}

Features on wafers manufactured by the semiconductor industry continue to decrease in size. Simulation is required to determine the dimensions of a feature from a microscope image when some of the dimensions are comparable to or significantly smaller than the wavelength of the light used in the microscope. Previously images of finite strips on a substrate have been approximated by those of infinite strips, which reduces to the solution of the two-dimensional scalar Helmholtz equations [1]. If this simpler approach no longer gives accurate results, we have to consider scattering of monochromatic plane waves of circular frequency $\omega$ by finite strips, which requires the solution of the three-dimensional Maxwell equations [2]. We use a generalization of the single-integral-equation formulation [3] that reduces the number of unknowns on the interfaces in three-dimensional problems such as a particle in a layer [4], a doublet of spheres [5], and a finite strip on a substrate [6], where we show the integral equations for the unknown tangential vector fields on the boundaries and the simulation of image formation. Here we show details of the scattering by a finite strip, such as the parameters defining the configuration of an oblique strip, the handling of the surface divergence term, and the reduction to algebraic equations, including self-patch contributions [7]. Most simulations of interest will be those of rectangular strips, as shown in Fig. 1, but to study deformations of such strips we need to be able to simulate oblique strips as shown in Fig. 2.

\section{Scattering by a rectangular strip on a substrate}

We choose the origin of the coordinate system on the substrate at the center of the strip, the $x$ - and $y$-axes parallel to sides of the strip and the $z$-axis perpendicular to the substrate. It suffices to give the width, height, and length to specify the shape of a rectangular strip. The surface $S_{1}$ is on the substrate outside the strip, $S_{2}$ is composed of the five faces of the strip, and $S_{3}$ is the base of the strip.

One of the four integral equations [6] for the unknown tangential vector fields $\vec{\chi}_{11}$, $\vec{\chi}_{12}, \vec{v}_{23}$, and $\vec{\chi}_{23}$ is

$$
\begin{aligned}
& {\left[\left(\frac{1}{2} \vec{\Im}+\overrightarrow{\mathrm{L}}_{21}^{\mathrm{t} 1}\right) \overrightarrow{\mathrm{M}}_{11}^{\mathrm{t} 1}+\overrightarrow{\mathrm{M}}_{21}^{\mathrm{t} 1}\left(\frac{1}{2} \overrightarrow{\mathfrak{\Im}}+\overrightarrow{\mathrm{L}}_{11}^{\mathrm{t} 1}\right)\right]\left\{\vec{\chi}_{11}\right\}+\left[\left(\frac{1}{2} \overrightarrow{\mathfrak{\Im}}+\overrightarrow{\mathrm{L}}_{21}^{\mathrm{t}^{1}}\right) \overrightarrow{\mathrm{M}}_{12}^{\mathrm{t} 1}+\overrightarrow{\mathrm{M}}_{21}^{\mathrm{t} 1} \overrightarrow{\mathrm{L}}_{12}^{\mathrm{t} 1}\right]\left\{\vec{\chi}_{12}\right\}} \\
& -\overrightarrow{\mathrm{L}}_{23}^{\mathrm{t}}\left\{\overrightarrow{\mathfrak{v}}_{23}\right\}-\overrightarrow{\mathrm{M}}_{23}^{\mathrm{t}}\left\{\vec{\chi}_{23}\right\}=-\left(\frac{1}{2} \overrightarrow{\mathfrak{\Im}}+\overrightarrow{\mathrm{L}}_{21}^{\mathrm{t} 1}\right)\left\{\hat{n}_{1} \times \Delta \overrightarrow{\mathrm{E}}^{\mathrm{h}}\right\}-\overrightarrow{\mathrm{M}}_{21}^{\mathrm{t} 1}\left\{\hat{n}_{1} \times \Delta \overrightarrow{\mathrm{H}}^{\mathrm{h}}\right\}
\end{aligned}
$$

where $\hat{n}_{1}$ is a unit normal to $S_{1}$ and the arguments of the functionals on the righthand side are differences of homogeneous fields in regions $V_{1}$ and $V_{2}$. The unit functional $\vec{\Im}$ is defined by $\vec{\Im}\{\vec{\chi}\}=\vec{\chi}$, and the other functionals are 


$$
\begin{gathered}
\overrightarrow{\mathrm{L}}_{j i}^{\mathrm{t} l}\left\{\vec{\xi}_{i}\right\}\left(\overrightarrow{\mathrm{r}}_{l}\right)=\hat{n}_{l}\left(\overrightarrow{\mathrm{r}}_{l}\right) \times \mathrm{P} \int_{S_{i}} \mathrm{~d} S^{\prime} \frac{\left(1-i k_{j} R_{l i}\right) \vec{\xi}_{i}\left(\overrightarrow{\mathrm{r}}_{i}{ }^{\prime}\right) \times \overrightarrow{\mathrm{R}}_{l i}}{4 \pi R_{l i}^{3}} \exp \left(i k_{j} R_{l i}\right), \\
\overrightarrow{\mathrm{M}}_{j i}^{\mathrm{t} l}\left\{\vec{\xi}_{i}\right\}\left(\overrightarrow{\mathrm{r}}_{l}\right)=-i \omega \mu_{j} \hat{n}_{l}\left(\overrightarrow{\mathrm{r}}_{l}\right) \times \mathrm{P} \int_{S_{i}} \mathrm{~d} S^{\prime}\left[\frac{\left(1-i k_{j} R_{l i}\right) \nabla_{s}^{\prime} \cdot \vec{\xi}_{i}\left(\overrightarrow{\mathrm{r}}_{i}{ }^{\prime}\right) \overrightarrow{\mathrm{R}}_{l i}}{4 \pi k_{j}^{2} R_{l i}^{3}}-\frac{\vec{\xi}_{i}\left(\overrightarrow{\mathrm{r}}_{i}^{\prime}\right)}{4 \pi R_{l i}}\right] \exp \left(i k_{j} R_{l i}\right) .
\end{gathered}
$$

The subindex $j$ refers to the region $V_{j}$ with constants $\varepsilon_{j}, \mu_{j}$, and $k_{j}=\omega \sqrt{ }\left(\varepsilon_{j} \mu_{j}\right)$, the subindex $i$ to $S_{i}$ where the integrations are performed and the tangential vector field $\vec{\xi}_{i}$ is defined, and the superindex $l$ to $S_{l}$ where the functional is evaluated at $\vec{r}_{l}$. The expression $\nabla_{s} \cdot \vec{\xi}_{i}$ stands for the surface divergence of $\vec{\xi}_{i}$. We note that $\overrightarrow{\mathrm{r}}_{l} \in S_{l}$, $\overrightarrow{\mathrm{R}}_{l i}=\overrightarrow{\mathrm{r}}_{l}-\overrightarrow{\mathrm{r}}_{i}{ }^{\prime}$, and $R_{l i}=\left|\overrightarrow{\mathrm{R}}_{l i}\right|$. The other integral equations have the same form.

\section{Scattering by an oblique strip on a substrate}

We specify the locations of the vertices $P_{1}$ to $P_{4}$ by giving the $x$ - and $y$-coordinates of these points on $S_{3}$. We have to find the coordinates for $P_{5}$ to $P_{8}$ so that the resulting faces are plane. One way to do this is to give the four angles the faces $S_{22}, S_{23}$, $S_{24}$, and $S_{25}$ form with $S_{1}$, namely $\beta_{22}, \beta_{23}, \beta_{24}$, and $\beta_{25}$. These angles are equal to $90^{\circ}$ for the rectangular strip. Four other edges are located along the intersections of these planes and we give the length of three of these edges, for instance, $\mathrm{L}_{15}, \mathrm{~L}_{26}$, and $\mathrm{L}_{37}$, which determine the coordinates of $P_{5}, P_{6}$, and $P_{7}$. The length of the fourth edge and the location of $\mathrm{P}_{8}$ are determined by the intersection of the this strip with the plane defined by the three other vertices. Simple vector equations define the normals and vertices of the oblique strip in terms of the chosen parameters.

\section{The surface divergence}

The surface divergence term in (3) can be approximated numerically by differences or it can be eliminated using integration by parts. The divergence theorem for a tangential vector function $\overrightarrow{\mathrm{F}}(\overrightarrow{\mathrm{r}})$ defined on a surface $S$ bounded by a curve C is [8]

$$
\int_{S} \mathrm{~d} S^{\prime}\left[\nabla_{s}^{\prime} \cdot \overrightarrow{\mathrm{F}}\left(\overrightarrow{\mathrm{r}}^{\prime}\right)+\kappa\left(\overrightarrow{\mathrm{r}}^{\prime}\right) \hat{n}\left(\overrightarrow{\mathrm{r}}^{\prime}\right) \cdot \overrightarrow{\mathrm{F}}\left(\overrightarrow{\mathrm{r}}^{\prime}\right)\right]=\oint_{C} \mathrm{~d} \overrightarrow{\mathrm{s}}^{\prime} \cdot \overrightarrow{\mathrm{F}}\left(\overrightarrow{\mathrm{s}}^{\prime}\right),
$$

where $\kappa$ is the mean curvature. For a closed surface $S$, the right-hand side vanishes and the integral is transformed to

$$
\begin{aligned}
& \overrightarrow{\mathrm{M}}(\overrightarrow{\mathrm{r}})=\oint_{S} \mathrm{~d} S^{\prime} \frac{(1-i k R) \nabla_{\mathrm{s}}^{\prime} \cdot \vec{\chi}\left(\overrightarrow{\mathrm{r}}^{\prime}\right) \overrightarrow{\mathrm{R}}}{4 \pi R^{3}} \exp (i k R) \\
& =\oint_{S} \mathrm{~d} S^{\prime} \vec{\chi}\left(\overrightarrow{\mathrm{r}}^{\prime}\right) \cdot\left[\frac{(1-i k R)\left(R^{2} \overrightarrow{\mathrm{I}}-3 \overrightarrow{\mathrm{R}} \overrightarrow{\mathrm{R}}\right)}{4 \pi R^{5}}+k^{2} \frac{\overrightarrow{\mathrm{R}} \overrightarrow{\mathrm{R}}}{4 \pi \mathrm{R}^{3}}\right] \exp (i k R) .
\end{aligned}
$$

The new integral is more singular than the original one. The surface element near the singularity at $\vec{r}$ is of the order of $R$, so that the most singular term of the integrand is of the order of $R^{-2}$. This integral is then regularized to

$$
\overrightarrow{\mathrm{M}}(\overrightarrow{\mathrm{r}})=\oint_{S} \mathrm{~d} S^{\prime}\left[\vec{\chi}\left(\overrightarrow{\mathrm{r}}^{\prime}\right)-\vec{\chi}(\vec{r})\right] \cdot\left[\frac{(1-i k R)\left(R^{2} \overleftrightarrow{\mathrm{I}}-3 \overrightarrow{\mathrm{R}} \overrightarrow{\mathrm{R}}\right)}{4 \pi R^{5}}+k^{2} \frac{\overrightarrow{\mathrm{R}} \overrightarrow{\mathrm{R}}}{4 \pi R^{3}}\right] \exp (i k R) .
$$


Use of this method has not been found to be advantageous and we prefer the numerical approximation discussed below.

\section{Reduction of integral equations to algebraic equations}

We use the point-matching method. We divide the interfaces into patches and the unknowns are defined at the centers and the equations are satisfied at these points. The interface $S_{1}$ of the substrate extends to infinity, but we choose a finite size with progressively larger patches as we approach the outer limits, where the fields and the unknowns are small and need not be determined precisely. Parallel edges of the strip are divided into the same number of equal segments, although it might be necessary to choose more segments near the edges to follow the divergences of the unknown fields, especially at the vertices. We divide the continuation of the edges into segments increasing in size by a geometric progression. This leads to some oddly shaped patches far from the strip, but we prefer this choice as opposed to having a varying number of patches in a given direction. There should be no patches with the centers on an edge because there some of the components of the electromagnetic fields and the unknown fields diverge. A possible selection of patches on $S_{1}$ and $S_{3}$ is shown in Fig. 3 for the rectangular strip and in Fig. 4 for the oblique strip.

We approximate the integrals by sums and assume that the integrands are constant over each patch. The integrand diverges for the self-patch and we evaluate the contributions by assuming that the unknown is approximately constant over that patch and that the integrations is carried out symmetrically as indicated by the $\mathrm{P}$ in front of the integral. The self-patch integrals for the rectangle $\Delta S$ of sides $\Delta x$ and $\Delta y$ are

$$
\overrightarrow{\mathrm{F}}(\overrightarrow{\mathrm{r}})=\mathrm{P} \int_{\Delta S} \mathrm{~d} S^{\prime} \frac{\overrightarrow{\mathrm{R}}}{4 \pi R^{3}}, \quad I(\overrightarrow{\mathrm{r}})=\int_{\Delta S} \mathrm{~d} S^{\prime} \frac{1}{4 \pi R},
$$

and we find

$$
\overrightarrow{\mathrm{F}}(\overrightarrow{\mathrm{r}})=0, \quad I(\overrightarrow{\mathrm{r}})=\Delta x \log \frac{\sqrt{(\Delta x)^{2}+(\Delta y)^{2}}+\Delta y}{\sqrt{(\Delta x)^{2}+(\Delta y)^{2}}-\Delta y}+\Delta y \log \frac{\sqrt{(\Delta x)^{2}+(\Delta y)^{2}}+\Delta x}{\sqrt{(\Delta x)^{2}+(\Delta y)^{2}}-\Delta x} .
$$

For the oblique strip we have to choose different local coordinate systems to make these evaluations. The derivatives of the surface divergence are approximated by second-order expansions. For instance, we have

$$
\partial \chi(u, v) / \partial u \approx[\chi(u+\Delta u, v)-\chi(u-\Delta u, v)] /(2 \Delta u)
$$

for interior patches of constant size and

$$
\partial \chi(u, v) / \partial u \approx[\mp 3 \chi(u, v) \pm 4 \chi(u \pm \Delta u, v) \mp \chi(u \pm 2 \Delta u, v)] /(2 \Delta u)
$$

at the left and right edges.

We solve the algebraic equations to find the unknown and use these values in the integrals that give the fields in any of the regions. A microscope image is then constructed from the fields in $V_{1}$ at a plane above the scatterer using equations from Fourier optics [6]. Far fields that are restricted to a given collection numerical aperture focused at a plane near the substrate determine the image. 


\section{References}

[1] E. Marx, "Images of Strips On and Trenches In Substrates," Appl. Opt. 46, 5571-5587 (2007).

[2] A. J. Poggio and E. K. Miller, "Integral Equation Solutions of ThreeDimensional Problems," in Computer Techniques for Electromagnetics, R. Mittra, Ed. Oxford: Pergamon, 1973.

[3] E. Marx, "Integral Equation for Scattering by a Dielectric," IEEE Trans. Antennas Propagat. 32, 166-172, 1984.

[4] E. Marx, "Integral Equation for Scattering by a Particle in a Layer" Digest of the IEEE Antennas and Propagation Society International Symposium, Montreal, 878-881 (1997).

[5] E. Marx, "Extinction Coefficients for Dielectric and Conducting Doublets of Spheres" Digest of the IEEE Antennas and Propagation Society International Symposium, Atlanta, 2198-2201 (1998).

[6] E. Marx, "Integral Equations for 3-D Scattering: Finite Strip on a Substrate," PIERS Online Vol. 5, No. 2, 133-136 (2009).

[7] E. Marx, "Self-Patch Integrals in Transient Electromagnetic Scattering," IEEE Trans. Antennas Propagat. 33, 763-767, 1985.

[8] Rutherford Aris, Vectors, Tensors, and the Basic Equations of Fluid Mechanics, p. 225, Dover, New York (1962).

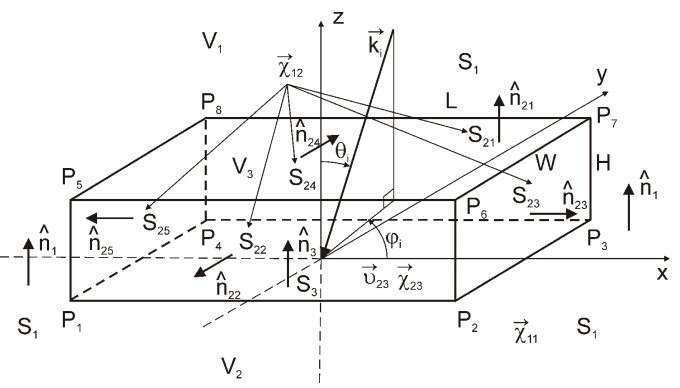

Fig. 1. Rectangular strip on a substrate.

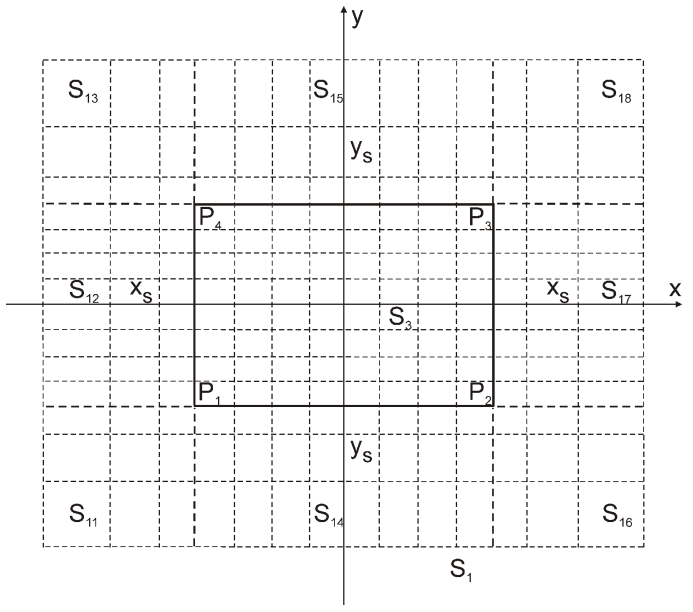

Fig. 3. Patches for the rectangular strip.

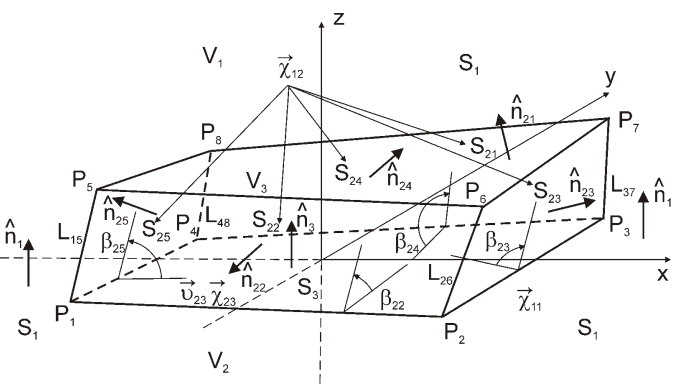

Fig. 2. Oblique strip on a substrate.

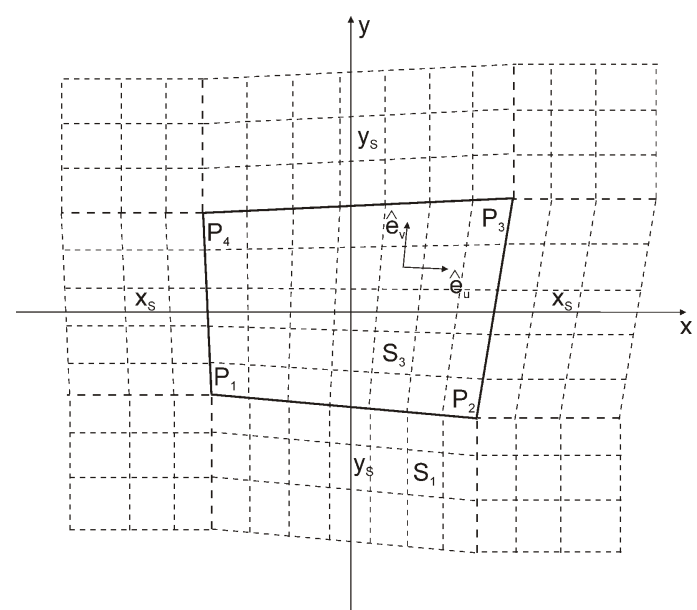

Fig. 4. Patches for the oblique strip. 